\title{
Recurrent Respiratory Papillomatosis Arising in Trachea not Affecting Larynx
}

\author{
Qingliang Xue and Jianxin Wang
}

\begin{abstract}
Recurrent respiratory papillomatosis (RRP) is an infrequent benign neoplasm commonly involving the upper respiratory tract with laryngeal predilection. The diagnosis and treatment of this disease are challenging due to its nonspecific clinical presentations and recurrent nature. We report here a rare juvenile case of RRP arising in the trachea without laryngeal lesions. Our experiences indicate that a high awareness of RRP and proper consideration in the right clinical context are prerequisites for early diagnosis, and a combination of multiple treatment modalities should be considered as a feasible treatment regimen.
\end{abstract}

Key words: recurrent respiratory papillomatosis, human papilloma virus, trachea, juvenile

(Inter Med 49: 1649-1651, 2010)

(DOI: 10.2169/internalmedicine.49.3443)

\section{Introduction}

Recurrent respiratory papillomatosis (RRP) is an exophytic and benign neoplasm with an incidence rate ranging approximately from 4 to 12 cases per 1,000,000 personyears (1). RRP can occur anywhere inside the respiratory tract; the most common lesion site is anatomically the larynx. According to the onset age of younger or older than age 13 years, RRP has been divided into juvenile-onset and adult-onset types. Two different types usually present distinct clinical courses. RRP arising in the trachea without a laryngeal lesion has been occasionally reported in adult cases (2). Here we report our experience of diagnosis and treatment in a juvenile case.

\section{Case Report}

A 12-year-old boy complained of cough and some white mucoid sputum for 4 months without fever, hemoptysis, wheezing, hoarseness or dyspnea. He was initially diagnosed as bronchitis and received anti-infective and symptomatic treatment. However, his symptoms did not improve. Two weeks before visiting our hospital, he started to present sporadic wheezing and underwent a thorax computed tomography (CT) examination. CT revealed multiple tumors arising from the posterior and right anterior wall of the mid-lower segment of trachea; the proximal and distal end of lesion site and the lung parenchyma were normal (Fig. 1). When the boy visited our hospital, outpatient bronchoscopy was performed. The bronchoscopic findings (Fig. 2) revealed multiple tumors encircling the wall of the trachea, approximately $6 \mathrm{~cm}$ below the glottis, and two-thirds of the tracheal lumen was obstructed. The tumors presented a cauliflowerlike shape without necrosis on the surface and were prone to hemorrhage after touching due to their fragile nature. The laryngeal area was normal. Biopsies were performed at several different lesion sites and histological findings revealed squamous papillomatosis (Fig. 3). He was thus admitted to hospital for further examinations and treatments.

Physical examinations on admission revealed inspiration prolongation and respiratory harshness on thorax auscultation. Serological study was negative for human immunodeficiency virus antibodies. In peripheral blood, hemoglobin were $136 \mathrm{~g} / \mathrm{L}$, platelets $261 \times 10^{9} / \mathrm{L}$, white blood cells $5.7 \times$ $10^{9} / \mathrm{L}$ with $42.9 \%$ neutrophils. The results of examinations of liver and kidney functions were normal. HPV11 DNA was identified by the method of nucleic acid hybridization in the biopsy samples (Fig. 4).

Because the lesions were extensive, it was dangerous and impossible to removal all of the lesions in a single time, and thus interval electrocautery procedures under local surface 


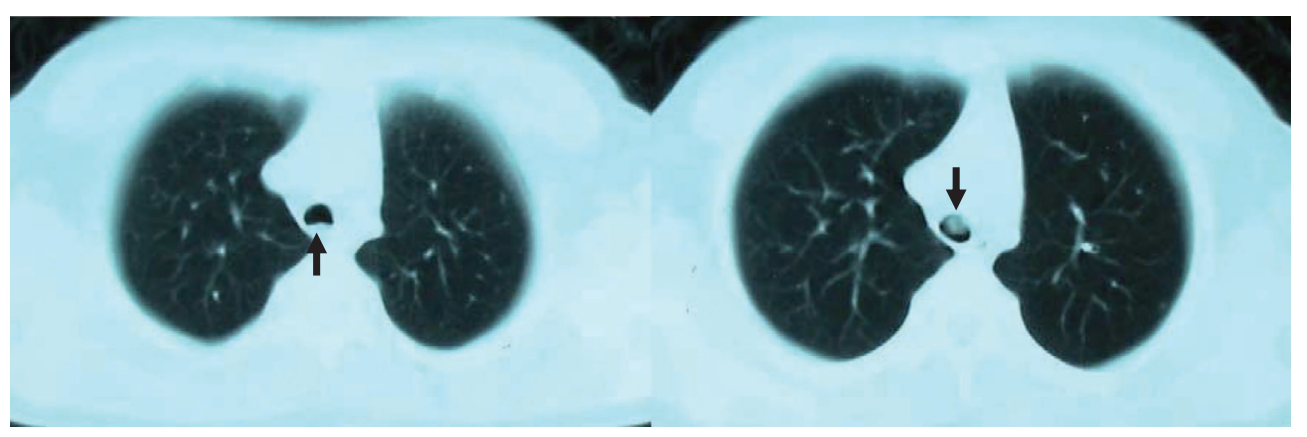

Figure 1. Thorax CT revealed multiple tumors which arose from the posterior and right anterior wall of trachea $(\boldsymbol{\top})$.

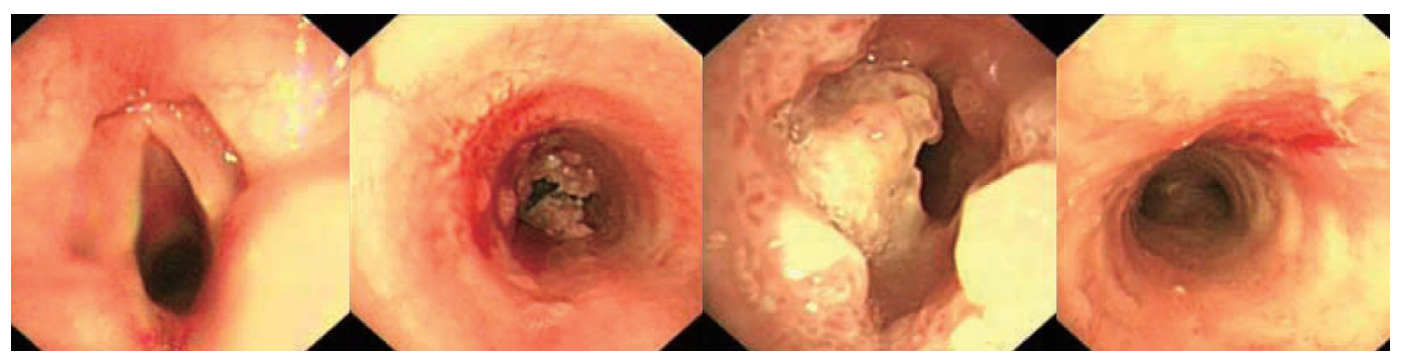

Figure 2. The bronchoscopic findings revealed multiple cauliflower-like tumors in the trachea without laryngeal lesions (left 3 figures). Tracheal structure recovered after two months of treatments (right figure).

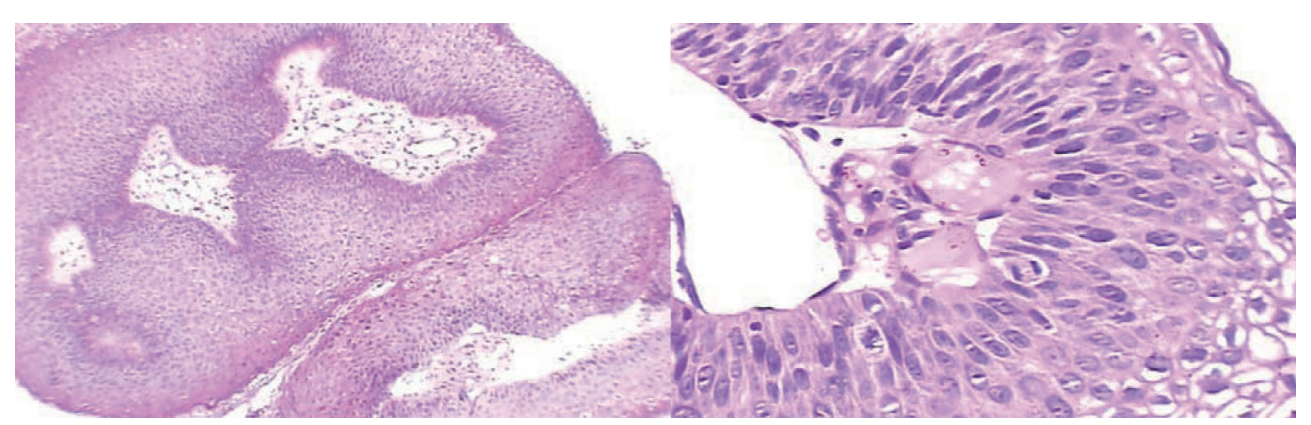

Figure 3. Histological findings revealed squamous papillomatosis (Hematoxylin and Eosin staining; left: original magnification $\times 40$, right: original magnification $\times 200$ ).

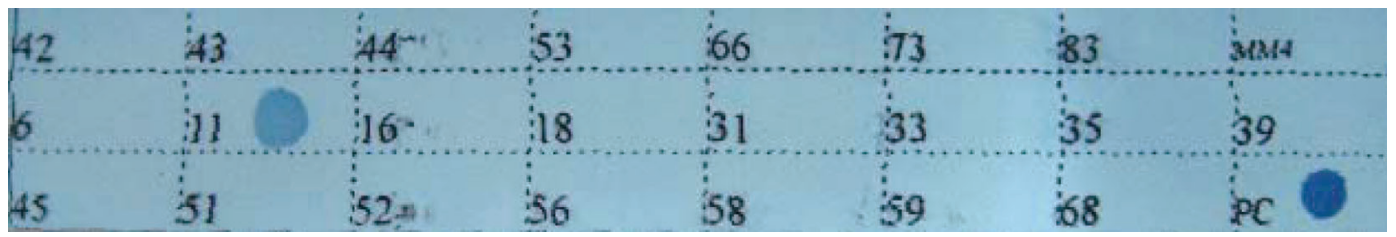

Figure 4. HPV11 DNA was identified in the biopsy sample by the method of nucleic acid hybridization (PC: positive control).

anesthesia were selected as the main treatment modality in order to lower medical fees. Equipments applied were the Olympus PSD-20 electrosurgical system and a flexible monopolar electrocautery probe (Olympus; Tokyo, Japan). The power output was set $25 \mathrm{~W}$ and the duration of electrocautery was less than $5 \mathrm{~s}$ per pulse. After five successive times of electrocautery on bronchoscope, most of the tumorous lesions in his trachea were removed. Meanwhile, he was ad- ministered the adjuvant treatments of acyclovir $(250 \mathrm{mg} / 8$ hours) and interferon (IFN)- $\alpha$ (100 wU/d). After two months of treatments, his clinical symptoms disappeared and the anatomic structure of the trachea recovered (Fig. 2). He had been followed up for one year and the lesions had not recurred and airway clearance was maintained. 


\section{Discussion}

Human papilloma virus (HPV) 11 was identified as the pathogen in this case. It is well known that RRP is primarily caused by HPV, of which HPV6 and HPV11 cause over $90 \%$ of cases (3). The human anogenital tract is a primary reservoir of HPV which is also the primary pathogen of anogenital warts. HPV can be transmitted from the mother's anogenital tract to infant's respiratory tract during delivery, which is a well established cause for juvenile-onset RRP. Iatrogenic transmission also deserves attention because HPV can survive on cryoprobe and in the plume generated by carbon dioxide laser. Additionally, extra-esophageal acid reflux disease should be regarded as a high risk factor for RRP $(4,5)$. RRP most commonly occurs in the larynx and lesions of the lower respiratory tract are usually due to the spreading of laryngeal lesions. The present case of juvenileonset tracheal papillomas without lesions in other areas of the airway, is rare. How this boy acquired HPV infection is unclear because there was no evidence of the boy suffering from gastroesophageal reflux disease or his mother suffering genital warts or iatrogenic transmission. Why the papillomatous lesions occurred only in the trachea in this case is also unclear. The immune status of the local site might be one of reasons.

The diagnosis of RRP is challenging due to its nonspecific clinical manifestations, especially for patients such as the present case. For early diagnosis, a high awareness of the variable presentations of RRP and proper consideration in the appropriate clinical context are prerequisites. Thorax CT and bronchoscopy in suspected patients would enable the diagnosis to be established early. Additionally, pulmonary function test might suggest some important clues for the diagnosis. However, because the boy performed pulmonary function test badly, we did not successfully obtain the spirogram before bronchoscopy.

To date, many treatment modalities for RRP have been tried, but none of them has been proven fully efficacious for a cure in all patients. Recurrence is common because HPV may persist latently in morphologically normal tissue adjacent to lesions. HPV11 infection and immunocompromised host are two high risk factors for recurrence. Surgical removal on endoscope is still the fundamental treatment to secure an adequate airway. Approximately 14\% of laryngeal Papillomatous lesions require tracheotomy to avoid lifethreatening airway obstruction (6). Antiviral and immunoregulation therapies are two important adjuvant treatments. HPV vaccine has been proven efficacious in preventing the HPV infections of the cervix and anogenital tract (7). However, the role of HPV vaccine in preventing RRP is not clear yet and it is less likely to be of therapeutic value (8). In this case, after the boy underwent the surgical removal via electrocautery and the adjuvant treatments of acyclovir and IFN- $\alpha$, the lesions were cured in a short time and had not recurred for one year, which indicated that the combination of multiple treatment modalities is one of the feasible treatment regimens.

\section{References}

1. Bishai D, Kashima H, Shah K. The cost of juvenile-onset recurrent respiratory papillomatosis. Arch Otolaryngol Head Neck Surg 126: 935-939, 2000.

2. Shibuya H, Kutomi T, Kujime K, Hara K, Hisada T. An adult case of multiple squamous papillomas of the trachea associated with human papilloma virus type 6. Intern Med 47: 1535-1538, 2008.

3. Steinbrook R. The potential of human papillomavirus vaccines. $\mathrm{N}$ Engl J Med 354: 1109-1112, 2006.

4. McKenna M, Brodsky L. Extraesophageal acid reflux and recurrent respiratory papilloma in children. Int J Pediatr Otorhinolaryngol 69: 597-605, 2005.

5. Pignatari SS, Liriano RY, Avelino MA, Testa JR, Fujita R, De Marco EK. Gastroesophageal reflux in patients with recurrent laryngeal papillomatosis. Braz J Otorhinolaryngol 73: 210-214, 2007.

6. Derkay CS. Task force on recurrent respiratory papillomas. A preliminary report. Arch Otolaryngol Head Neck Surg 121: 13861391, 1995.

7. Hsueh PR. Human papillomavirus, genital warts, and vaccines. J Microbiol Immunol Infect 42: 101-106, 2009.

8. Vandepapeliere P, Barrasso R, Meijer CJ, et al. Randomized controlled trial of an adjuvanted human papillomavirus (HPV) type 6 L2E7 vaccine: infection of external anogenital warts with multiple HPV types and failure of therapeutic vaccination. J Infect Dis 192: 2099-2107, 2005.

(C) 2010 The Japanese Society of Internal Medicine http://www.naika.or.jp/imindex.html 